\title{
Studies of Dark Current Reduction in InAsSb Mid-Wave Infrared HOT Detectors through Two Step Passivation Technique
}

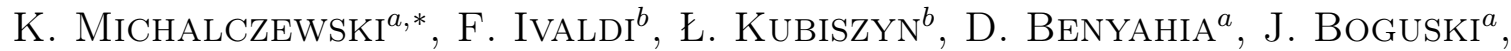 \\ A. KęBeOWski ${ }^{b}$, P. MARtyniuk ${ }^{a}$, J. Piotrowski ${ }^{b}$ And A. Rogalski ${ }^{a}$ \\ ${ }^{a}$ Institute of Applied Physics, Military University of Technology, S. Kaliskiego 2, 00-908 Warsaw, Poland \\ ${ }^{b}$ Vigo System S.A., Poznańska 129/133, 05-850 Ożarów Mazowiecki, Poland
}

\begin{abstract}
We report on the investigation of the surface leakage current for $\operatorname{InAs}_{1-x} \mathrm{Sb}_{x}(x=0.09)$ high operation temperature photodiode grown on GaAs substrate in accelerated short-term stability test. The electrochemical passivation technique was proposed to modify the mesa sidewalls properties and obtain anodic sulphur coating covered by SU-8 negative photoresist. The electrical behavior of sulphur anodic film, SU-8 photoresist, and unpassivated devices was compared for devices in variable area diode array test. The surface resistivity for anodic sulphur film, SU-8 and unpassivated devices are equal to $1080,226,10200 \mathrm{k} \Omega \mathrm{cm}$, respectively, at $150 \mathrm{~K}$ and 1340 $429,2870 \mathrm{k} \Omega \mathrm{cm}$, respectively, at $150 \mathrm{~K}$ after an exposure of $20 \mathrm{~h}$ to atmosphere at $373 \mathrm{~K}$. The Auger recombination process was evaluated as the main mechanism of diffusion current in HOT devices.
\end{abstract}

DOI: 10.12693/APhysPolA.132.325

PACS/topics: 73.61.Ey, 73.25.+i, 82.45.Cc

\section{Introduction}

The application associated with gas detection and infrared imaging in the spectral range of $3-5 \mu \mathrm{m}$ have stimulated considerable interest in the development of infrared (IR) detectors especially with InAsSb alloy.

It has been known that this material has got a number of advantages as wide spectral range due to composition, low electron effective mass, and high mobility at room temperature [1]. Implementation of such alloy in barrier devices grown on GaAs substrate is a convenient way to obtain cheap high operation temperature (HOT) devices which could compete with $\mathrm{HgCdTe}$ technology.

One of the limiting factor of all infrared devices is leakage current. Up to date there is still discussion about the importance of passivation and influence of surface leakage current in III-V materials technology in long term stability.

It is believed that surface leakage current is reduced by proper device architecture (unipolar barrier [2]) and processing $[2,3]$. Craig et al. proposed special mesas processing in their $\mathrm{nBn}$ devices which leads to a high shunt resistance. Unfortunately, all structures were grown on costly GaSb substrates and no long term stability test had been made.

In order to produce cheap infrared focal plane arrays (IR FPAs) production devices it is recommended to utilize GaAs substrate. GaAs has better structural, optical, and thermal properties than GaSb. In addition, these substrates are more affordable and available as large size

\footnotetext{
* corresponding author; e-mail: krystian.michalczewski@wat.edu.pl
}

"epi-ready" wafers up to 6 inch in diameter. Transparent GaAs substrate allows for the backsided device illumination and fabrication of monolithic optical immersion.

During the processing, the devices with GaAs substrate, the barrier is removed in etching process and mesas sidewalls are exposed to aggressive chemicals and atmosphere. It results in saturating of dangling bond and formation of secondary compounds which create additional interfacial states. Interfaces states located within band-gap alleviate the carrier tunneling, inducting surface recombination and contribute to trap-assisted tunneling dark current [4].

The X-ray photoelectron spectroscopy (XPS) measurements $[5,6]$ showed that after processing, elemental arsenic and antimony are distributed through the native oxide. It causes the formation of conduction channels parallel to the interface which could lead to an increase of the overall dark current.

Numerous passivation techniques are available such as dielectric deposition, over growth with wide-gap materials, SU-8 passivation, atomic modification of structure by sulphur or fluorine.

All of these techniques require additional special equipments or advanced procedures of preparation and are less effective or even destructive when they are utilized singly [7]. One of the most simple way to passivate the III-V materials is to treat with sulphur or fluorine chemistry. However, Banerjee et al. [8] reported that sulphur coating is sufficient only in short-term stability and suitable capping layer is necessary. The other way is to use SU-8 negative photoresist which after the hard baking is mechanically and chemically stable [9]. In this case it is hard to maintain surface free from native oxide before SU-8 cover. 
In this paper we report on a passivation procedure based on 2 step technique (SU-8 coating after S-anodic treatment) and we discuss the surface leakage current in InAsSb HOT devices. To our best knowledge, such investigation of surface leakage current in $\mathrm{HOT}$ InAsSb grown on GaAs substrate has not been reported.

\section{Experiment}

The InAsSb photodiode was grown by a RIBER Compact 21-DZ solid source molecular beam epitaxy (MBE) system, on GaAs (001) substrates. The system is equipped with double filament effusion cells for gallium (Ga) and indium (In), and with valved cracker cells for arsenic (As) and antimony ( $\mathrm{Sb}$ ). $\mathrm{As}_{2}$ and $\mathrm{Sb}$ have been used. The manipulator thermocouple was used to monitor the substrate temperature.

The ICP-RIE process (Sentech Si 500) was utilized to produce mesas profile. The $\mathrm{SiCl}_{4}$ based plasma etching was used to receive uniform smooth morphology and etch depth $3.5 \mu \mathrm{m}$ which corresponds to the middle of the bottom contact layer of the detector. After etching sample was degreased and cleaned in acetone and TCE. Than the sample was cut into 3 pieces. The first piece (sample 1) was left as-etched without any passivation. The second piece (sample 2) was covered only with SU-8 negative photoresist. The third one (sample 3) was treated by electrochemical passivation $(\mathrm{ECP})$ by $\left(\mathrm{NH}_{4}\right)_{2} \mathrm{~S}$ prior to SU-8 cover. The ECP cell consists of the sample (anode), a platinum mesh electrode (cathode) and electrolyte $\left(20 \%\left(\mathrm{NH}_{4}\right)_{2} \mathrm{~S}\right.$ in $\left.\mathrm{H}_{2} \mathrm{O}\right)$. During the anodization a DC current density of $3 \mathrm{~mA} / \mathrm{cm}^{2}$ was supplied for $1 \mathrm{~min}$. After $1 \mathrm{~min}$ the sample was dried with $\mathrm{N}_{2}$ and mesas sidewalls were covered by SU-8. The mechanism of reaction with $\left(\mathrm{NH}_{4}\right)_{2} \mathrm{~S}$ is described in Banerjee work [8]. Prior to the Au-contact deposition the anodic film from the top and the bottom contact layer were removed with diluted $\mathrm{HCl}$ solution. The bottom and the top Au contact layers were deposited by electrochemical deposition (ECD). The set of 5 rounded mesas was produced with a diameter respectively 100, 200, 300, 400, $500 \mu \mathrm{m}$. The effectiveness of the passivation treatment was evaluated by the measurement of the temperature dependence of dynamic impedance-area product at zero bias and the dark current densities. Long term stability (st) of passivation layers was evaluated by accelerated tests at $100^{\circ} \mathrm{C}$ for $20 \mathrm{~h}$.

\section{Results and discussion}

The backside illuminated InAsSb diode, with unipolar electron barrier $\left(\mathrm{AlAs}_{0.1} \mathrm{Sb}_{0.9}\right)$ was optimized for methane detection at $230 \mathrm{~K}$ zero bias operation. Figure 1 shows the spectral response of unpassivated device under different bias. This devices show detectivity $1.0 \times 10^{10}$ Jones at $230 \mathrm{~K}$ under zero bias. The theoretical value of detectivity utilizing monolithic optical immersion lens is estimated to be more than $1.0 \times 10^{11}$ Jones.

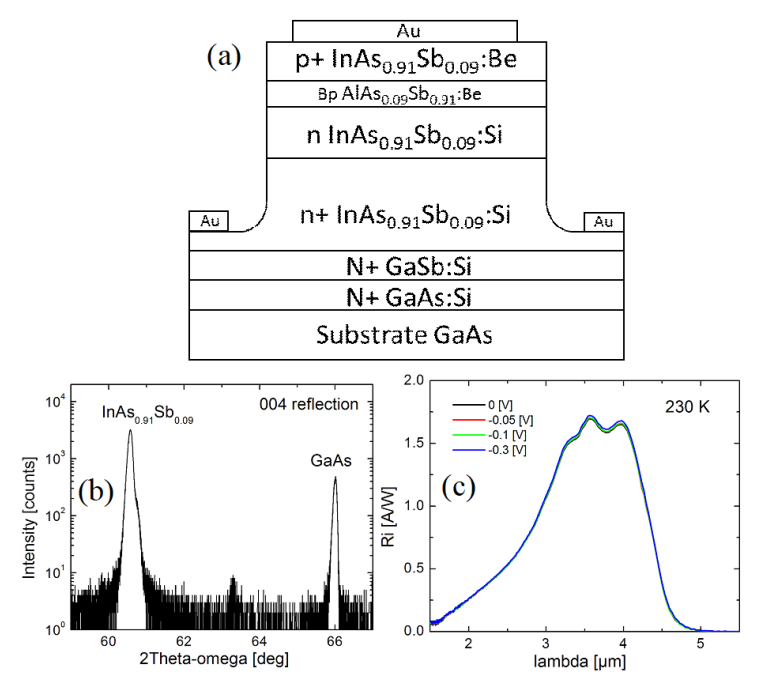

Fig. 1. Architecture (a), HRXRD spectra (b), spectral response of the photodetector at different bias (c) of In $\mathrm{AsSb}$ diode.

Figure 2 shows the dark current versus applied bias, measured by Probe Station analyzer, for passivated and unpassivated devices with a mesa diameter of $100 \mu \mathrm{m}$, before and after annealing at a set of temperatures. All passivated devices showed an increase of dark current compared to the unpassivated device.
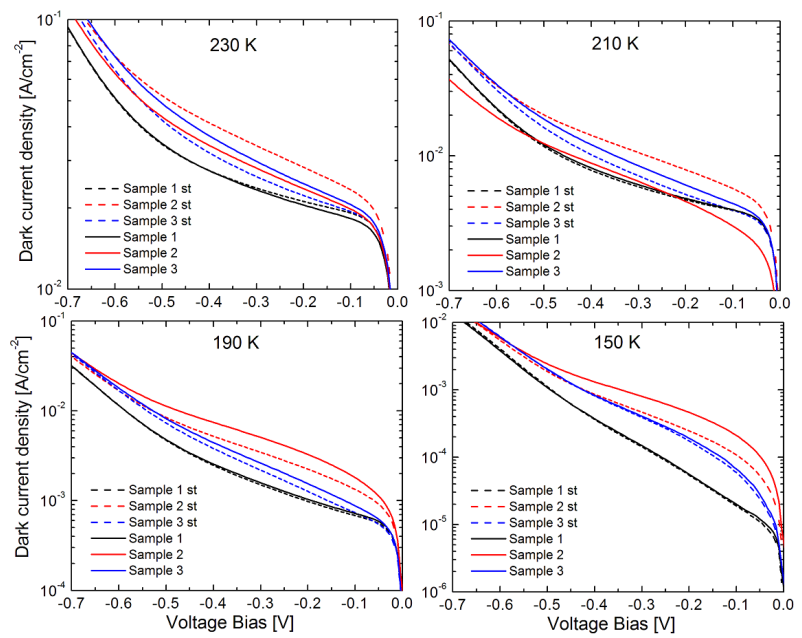

Fig. 2. Dark current density vs. applied bias characteristics of mesa diameter $100 \mathrm{\mu m}$ for different passivation techniques and after $20 \mathrm{~h}$ in $100^{\circ} \mathrm{C}$.

The dark current increased by a factor of 2 and 4 respectively for sample 2 and 3 compared to sample 1 for bias $-0.2 \mathrm{~V}$ at $230 \mathrm{~K}$. For sample 1 and sample 1 st there was no increase of the dark current at whole temperature range. That means that at higher temperature (minimum TE-cooler temperature) the surface leakage current is not dominant for InAsSb devices and it is suppressed mostly by unipolar electron barrier. For sample 2, 3, 2 st, 
3 st we observed an increase of surface leakage current contribution to dark current. At $150 \mathrm{~K}$ the difference in surface leakage current is the biggest.

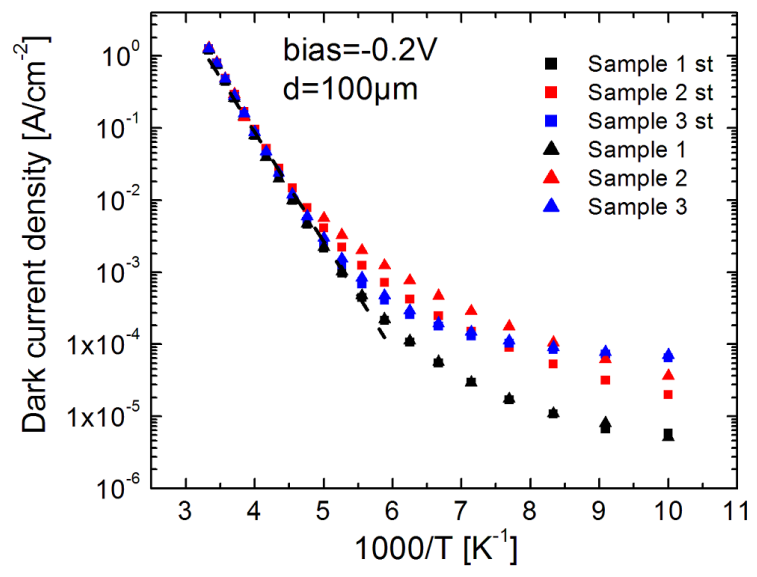

Fig. 3. Temperature dependence of dark current.

The SU-8 samples had the higher dark current densities which could be caused by the process of development of the SU-8 photoresist with standard procedure and the use of primer before the SU-8 covering. Similar problem was described in the passivation of bulk InAs diode. Ker in [10] described the special procedure of developing SU8 photoresist at lower baking temperature resulting in lower dark current. For sample 3 and 3 st the increase of surface leakage current was lower. For 3 st at each temperature the surface leakage current was reduced after annealing. We can expect that annealing at higher temperature/time could reduce dark current even more.

The Arrhenius plot of the dark current density (Fig. 3) shows that at higher temperature for all samples the dark current was dominated by the diffusion current. For sample 1 st and 3 st the surface leakage current did not increase after annealing. Only in sample 2 st we observed a small increase of the total surface leakage current. Below $180 \mathrm{~K}$, the mechanism of generation of the dark current is changed. The estimated value of $E_{a}=0.28 \mathrm{eV}$ was almost equal to $E_{g}$ of the absorber layer which means that the diffusion current is mostly generated by the Auger recombination process.

Figure 4 shows a plot of the inverse of the dynamic resistance-area product $\left(R_{0} A\right)$ at zero bias vs. the perimeter to area ratio. The surface dependence of the inverse of the dynamic resistance-area at zero bias $1 /\left(R_{0} A\right)$ can be approximated as

$$
\frac{1}{R_{0} A}=\frac{1}{R_{0} A_{\text {bulk }}}+\frac{1}{r_{\text {surface }}} \frac{P}{A}
$$

where $\left(R_{0} A\right)_{\text {bulk }}$ is the bulk $R_{0} A$ contribution in $\left[\Omega \mathrm{cm}^{2}\right]$, $r_{\text {surface }}$ is the surface resistivity $[\Omega \mathrm{cm}], P$ is the diode perimeter and $A$ is the diode area.

For the diode of infinite size $\left(\frac{P}{A}=0\right), R_{0} A$ given by Eq. (1) yields the $\left(R_{0} A\right)_{\text {bulk }}$ value of the material, independent of the surface effect. The slope of the function
TABLE I

Surface resistivity and $\left(R_{0} A\right)_{b u l k}$ values at $150 \mathrm{~K}$ extracted from Eq. (1).

\begin{tabular}{c|c|c|c|c}
\hline \hline \multicolumn{2}{c|}{} & sample 1 & sample 2 & sample 3 \\
\hline $\begin{array}{c}\text { surface } \\
\text { resistivity } \\
{[\Omega \mathrm{cm}]}\end{array}$ & $\begin{array}{c}\text { after } \\
\text { processing }\end{array}$ & $1.02 \times 10^{7}$ & $2.26 \times 10^{5}$ & $1.08 \times 10^{6}$ \\
\cline { 2 - 5 }$\left(20 \mathrm{~h} 100^{\circ} \mathrm{C}\right.$ & $2.87 \times 10^{6}$ & $4.23 \times 10^{5}$ & $1.34 \times 10^{6}$ \\
\hline$\left(R_{0} A\right)_{\text {bulk }}$ & $\begin{array}{c}\text { after } \\
\text { processing }\end{array}$ & $3.39 \times 10^{3}$ & $3.60 \times 10^{3}$ & $3.46 \times 10^{3}$ \\
\cline { 2 - 5 }$\left[\Omega \mathrm{cm}^{2}\right]$ & $20 \mathrm{~h} 100^{\circ} \mathrm{C}$ & $3.45 \times 10^{3}$ & $3.62 \times 10^{3}$ & $3.48 \times 10^{3}$
\end{tabular}

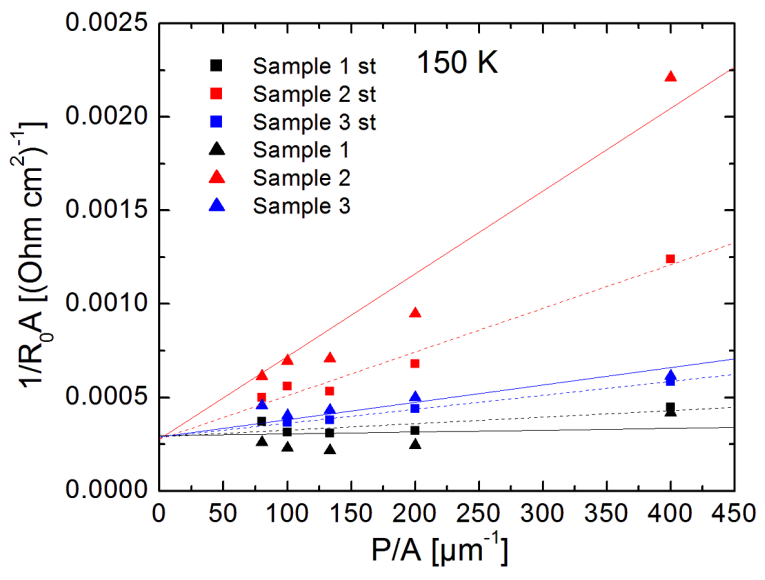

Fig. 4. $1 /\left(R_{0} A\right)$ as a function of $P / A$ at $150 \mathrm{~K}-$ for different passivation technique and after $20 \mathrm{~h}$ in $100^{\circ} \mathrm{C}$.

given by Eq. (1) is directly proportional to the surfacedependent leakage current of the diode. Higher values of the surface resistivity indicate the weaker dependence of the diode characteristic on the surface effects.

The extracted values of the surface resistivity and $\left(R_{0} A\right)_{\text {bulk }}$ at $150 \mathrm{~K}$ are presented in Table I. The highest values of surface resistivity were achieved in sample 1. This means that our approach of passivation mesa sidewalls was not sufficient and it increases the surface leakage current. It is worth to emphasize that before and after accelerated stability test the contribution of the surface effect on diode characteristic was very low even at $150 \mathrm{~K}$. For sample 3 and sample 2 a small increase of surface resistivity at the same time was observed. It could be correlated with the lowering of the surface leakage current observed in Fig. 2. Our results are an evidence that in III-V bulk barrier devices operating in HOT conditions, the surface leakage current might not be as important as in cryogenic temperature $(77 \mathrm{~K})$.

\section{Summary and conclusions}

We have demonstrated the result of passivated and unpassivated InAsSb barrier diode on GaAs substrate operating at $\mathrm{HOT}$ conditions.

We obtained the devices with a good crystal quality grown on GaAs substrate, where the diffusion current is 
generated mainly by the Auger recombination process at higher temperatures. The highest values of surface resistivity were achieved for unpassivated sample 1. This means that our proposed two step passivation technique was not sufficiently enough. In both sample 2 and 3 , we observed an increase of the surface leakage current. Future investigation will be proceeded to see the influence of annealing of sample 2 st and 3 st on reduction of the leakage current. It might be that annealing at higher temperature/time could reduce the dark current even more. Taking into account all results it could be concluded that the passivation layer in bulk barrier diode might play a main role as mechanical barrier to atmosphere, production technology, and might be crucial for long-term stability studies. Future investigation of the influence of the annealing process in the reduction of dark current will be done for passivated samples.

\section{Acknowledgments}

The authors would like to acknowledge the support by Polish National Science Centre: grant no. PRELUDIUM/UMO-2015/19/N/ST7/01508 and OPUS/UMO-2015/17/B/ST5/01753.

\section{References}

[1] A. Rogalski, Infrared Detectors, 2nd ed., CRC Press, 2011.

[2] D.E. Sidor, G.R. Savich, G.W. Wicks, J. Electron. Mater. 45, 4663 (2016).

[3] A.P. Craig, M.D. Thompson, Z.B. Tian, S. Krishna, A. Krier, A.R.J. Marshall, Semicond. Sci. Technol. 30, 105011 (2015).

[4] E.A. Plis, M.N. Kutty, S. Krishna, Laser Photon. Rev. 7, 45 (2013).

[5] G. Hollinger, R. Skheyta-Kabbani, M. Gendry, Phys. Rev. B 49, 11159 (1994).

[6] G.P. Schwartz, Thin Solid Films 103, 3 (1983).

[7] J. Hoffmann, T. Lehnert, D. Hoffmann, H. Fouckhardt, Semicond. Sci. Technol. 24, 065008 (2009).

[8] K. Banerjee, S. Ghosh, E.A. Plis, J. Electron. Mater. 39, 2210 (2010).

[9] H.S. Kim, E.A. Plis, N. Gautam, S. Myers, Y. Sharma, L.R. Dawson, S. Krishna, Appl. Phys. Lett. 97, 143512 (2010).

[10] P.J. Ker, Ph.D. Thesis, University of Sheffield, 2012. 